\title{
Electrospun 4th-Generation Solid Dispersions of Poorly Water-Soluble Drug Utilizing Two Different Processes
}

\author{
Zhu Zhang, ${ }^{1}$ Wenbing Li ${ }^{D},{ }^{2}$ Guanghua Wang, ${ }^{2}$ Yang-Lu Qu, ${ }^{3}$ and Deng-Guang Yu $\mathbb{D}^{3}$ \\ ${ }^{1}$ Department of Internal Medicine, The Hospital of Wuhan University of Science \& Technology, \\ Wuhan 430081, China \\ ${ }^{2}$ School of Chemistry \& Chemical Engineering, Wuhan University of Science \& Technology, \\ Wuhan 430081, China \\ ${ }^{3}$ School of Materials Science \& Engineering, University of Shanghai for Science \& Technology, \\ Shanghai 200093, China \\ Correspondence should be addressed to Wenbing Li; liwenbing@126.com and Deng-Guang Yu; ydg017@usst.edu.cn
}

Received 2 October 2017; Accepted 16 January 2018; Published 26 February 2018

Academic Editor: Zeeshan Khatri

Copyright ( 2018 Zhu Zhang et al. This is an open access article distributed under the Creative Commons Attribution License, which permits unrestricted use, distribution, and reproduction in any medium, provided the original work is properly cited.

\begin{abstract}
Different from traditional solid dispersion (SD) for improving the dissolution rates of poorly water-soluble drugs, the upgraded 4th SD was developed to furnish a drug sustained-release profile. In this work, two different kinds of 4th SDs were fabricated using two electrospinning processes. One is a ternary SD (nanofibers F2) that consisted of ethyl cellulose (EC), polyethylene glycol 1000 (PEG), and tamoxifen citrate (TAM) from a modified coaxial process, and the other is a binary SD (nanofibers F1) which is comprised of EC and TAM from a single-fluid blending process. Scanning electronic microscopic observations demonstrated that F2 $(330 \pm 50 \mathrm{~nm})$ showed a better quality than F1 $(870 \pm 230 \mathrm{~nm})$ in terms of size and size distribution although both of them had a smooth surface morphology and a cross section. X-ray diffraction patterns verified that both SDs were amorphous nanocomposites owing to the favorable secondary interactions among these components, as suggested from the results of FTIR. In vitro dissolution experiments indicated that F2 could furnish an improved drug sustained-release characteristics compared to F1, exhausting all the contained TAM and having weaker leveling-off late release. The molecular behaviors of drug sustained-release from the binary 4th SD were suggested. The protocols reported here paved an alternative way for developing novel functional nanomaterials for effective delivery of poorly water-soluble drugs.
\end{abstract}

\section{Introduction}

Traditionally, solid dispersion (SD) is a popular approach for enhancing the dissolution rates of insoluble drugs [1]. Numerous publications have reported the applications of soluble polymer excipients (such as polyvinylpyrrolidone, poly(ethylene oxide), poly(vinyl alcohol), gelatin, and some other natural products, e.g., water-soluble polysaccharide) as matrices for developing SDs using a wide variety of advanced methods $[2,3]$. However, the concept of SD for enhancing the dissolution rates of insoluble drugs is moving forward fast to adjust drug controlled release profiles, and thus the 4th
SD has drawn increasing attention today $[4,5]$. Regardless of the generations of SD (1st to 4th), the double directions for the further developments are obvious. One is to reduce the final products to the nanoscale to make full use of the huge surface area, and the other is the combined usage of different types of drug carriers as matrices to highly disperse the drug molecules. As for the 4 th $\mathrm{SD}$, a drug distribution in the insoluble drug carrier on a molecular scale often means a designable drug sustained-release profile [6].

Among different nanotechnologies that have been introduced in generating $\mathrm{SD}$, electrospinning is one of the 
hottest ones owing to its effectiveness, facile implementation, low cost, and the properties of the electrospun nanoproducts [7-11]. The first publication of electrospun medicated nanofibers was essentially about a drug sustained-release 4th SD, although the authors did not directly point out in the text [12]. Recently, on one hand, this technology has quickly developed from the treatment of a singleblending solution to simultaneous treatments of double fluids in organized formats, such as coaxial or side-by-side because of the usefulness of core-shell and Janus nanostructures in designing advanced functional nanomaterials $[13,14]$. On the other hand, the treated fluids are expanded from electrospinnable solutions to the combined usages of them with unelectrospinnable liquids, such as the modified coaxial/triaxial and side-by-side electrospinning [15-17]. Nonetheless, SDs from a single-fluid blending and coaxial processes are still the mainstream in this field, which also hold the great promises to be scaled up for productions [18].

During the past over ten years, the single-fluid blending electrospinning process has been both frequently utilized to prepare 2nd and 3rd SDs from amorphous water-soluble candidates for enhancing drug dissolution rates and also frequently exploited to create 4 th SDs from insoluble or degradable polymers for providing a sustained-release profile [19]. No matter what cases they are, most of the reports focused on the SDs with one guest drug distributed among one host polymeric excipient, whose chemical and physical properties are relied on to adjust the drug release mechanics for a designed drug controlled release profile and also an improved therapeutic effect $[3,4]$.

From a standpoint of materials science and engineering, SDs (particularly those polymer-based SDs) are essentially medicated nanocomposites [6]. The combined usage of multiple excipients with different properties can often generate new promises for drug delivery. One example is the combination of water-soluble polymeric carrier with surfactant in the 3rd SDs, which forms a solid cock-tail of poorly water-soluble drug for fast disperse and therapeutic action [19]. This strategy can also be applied in developing new types of 4 th SDs, which are SDs providing both solubility enhancement and extended release in a controlled manner of the loaded poorly water-soluble drugs [4].

In this work, we investigated the combined usages of ethyl cellulose (EC) and polyethylene glycol 1000 (PEG) for adjusting the release rates of tamoxifen citrate (TAM), whose sustained-release profile is highly desired by the patients [20]. A traditional blending electrospinning and a modified coaxial electrospinning were utilized to prepare the binary and ternary 4 th SDs, respectively. Their morphology, physical state, properties, and functional performances were compared in detail. EC and PEG are polymeric excipients frequently utilized in pharmaceutical industries. The former is an insoluble natural polymer with fine filament-forming property and often exploited for drug sustained-release. The latter is an soluble oligomer and also a surfactant, which has no electrospinnability [21]. TAM is a typical poorly water- soluble anticancer active ingredient that is commercially available in several orally administered forms and dosages.

\section{Materials and Methods}

2.1. Materials. EC $(6 \sim 9 \mathrm{mPa} \cdot \mathrm{s})$ was achieved from Shanghai Yunhong Pharm Aid \& Technol Co., Ltd. (Shanghai, China). TAM (>99\%) was obtained from Wuhan Beika Biological Pharm Co., Ltd. (Wuhan, China). PEG 1000, dichloromethane, methylene blue, and anhydrous ethanol were obtained from Shanghai Chemical Reagents Co., Ltd. (Shanghai, China). All chemicals (analytical reagents) were utilized as received.

2.2. Electrospinning. The preparations of nanofibers were carried out on a coaxial electrospinning system, which consisted of a high voltage generator (ZGF60 kV/2 mA, Wuhan Hua-Tian Co., Ltd., Hubei, China), two KDS100 syringe pumps (Cole-Parmer, USA), and a collector. The concentric spinneret was homemade and the collector was prepared by wrapping aluminum foil around a cardboard.

After some preliminary experiments, two 4th SDs were prepared. One is nanofibers F1 from a single-fluid blending process. A working solution composed of 25\% EC and 5\% TAM (all in w/v) in a mixed solvent (ethanol:dichloromethane, 1:1 in volume) was treated under the following experimental conditions: the applied voltage was $12 \mathrm{kV}$, the fiber-collected distance was $15 \mathrm{~cm}$, and the flow rate was $1.0 \mathrm{~mL} / \mathrm{h}$.

The other is nanofibers F2 from a modified coaxial process. A blending solution consisting of $25 \% \mathrm{EC}$, 5\% PEG, and 5\% TAM (all in $\mathrm{w} / \mathrm{v}$ ) in a mixed solvent (ethanol: dichloromethane, 1:1 in volume) was utilized as the core working liquid $(5 \mu \mathrm{g} / \mathrm{mL}$ methylene blue was added to the core solution for investigating the experimental conditions). The sheath working fluid was pure ethanol. The experimental conditions are listed as follows: the applied voltage was $12 \mathrm{kV}$, the fiber-collected distance was $15 \mathrm{~cm}$, the core fluid flow rate was $1.0 \mathrm{~mL} / \mathrm{h}$, and the sheath ethanol flow rate was $0.3 \mathrm{~mL} / \mathrm{h}$. A PowerShot A640 camera (Tokyo, Japan) was exploited to monitor the electrospinning processes.

2.3. Morphology. The surface morphology of the nanofibers was evaluated using a Quanta 200 FEG scanning electron microscope (SEM, FEI, USA). The cross-sectional samples were prepared by manually breaking a nanofiber sheet after it was immersed in the liquid nitrogen for over 15 mins. All the specimens were gold sputter-coated before they were put into the SEM machine. ImageJ software (NIH, Bethesda, MD, USA) was exploited to determine the diameters of nanofibers at more than one hundred places.

2.4. Physical State and Compatibility. X-ray diffraction (XRD) tests were conducted on a Bruker X-ray Powder Diffractometer Bruker-AXS with $\mathrm{Cu} \mathrm{K} \alpha$ radiation (Karlsruhe, Germany) under an applied voltage of $40 \mathrm{kV}$ and a 


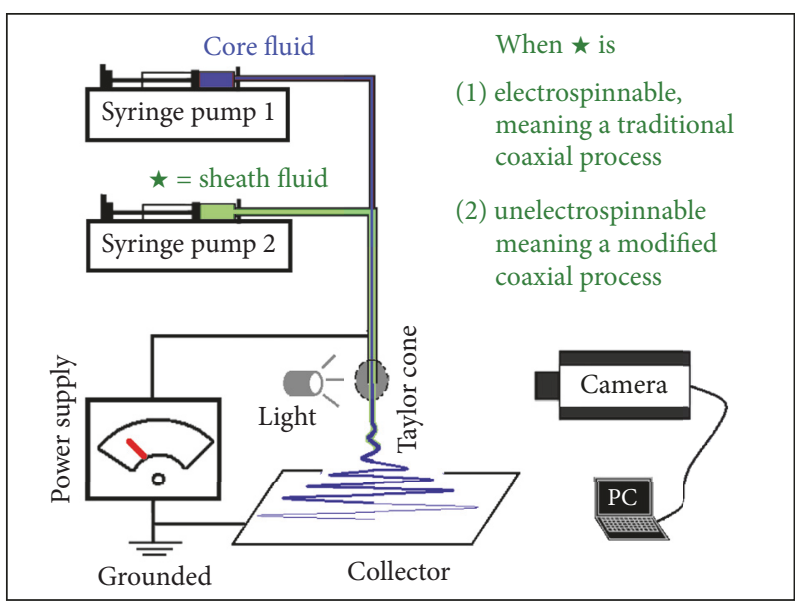

(a)

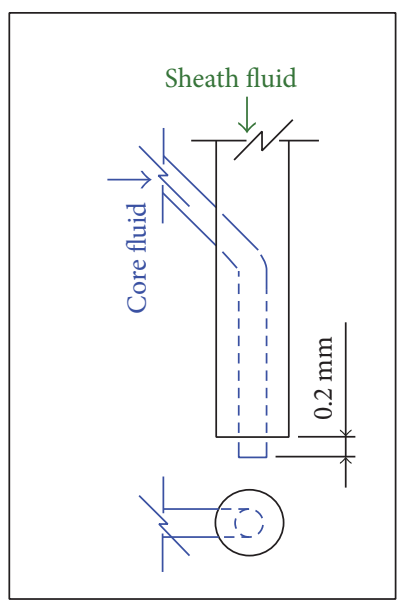

(b)

Figure 1: Diagrams of the coaxial electrospinning (a) and the concentric spinneret (b).

current of $30 \mathrm{~mA}$ and between a $2 \theta$ range of $5^{\circ}$ to $60^{\circ}$. The raw EC/TAM powders, PEG thin slices, and their electrospun 4th SDs were subjected to attenuated total reflectanceFourier transform infrared (ATR-FTIR) analyses using a Spectrum 100 FTIR Spectrometer (PerkinElmer, Billerica, USA).

2.5. In Vitro Dissolution Tests. A RCZ-8A apparatus (TianJin University Radio Factory, Tian-Jin, China) was exploited to conduct the in vitro dissolution tests. $0.18 \mathrm{~g}$ nanofibers F1 and $0.21 \mathrm{~g}$ nanofibers F2 (containing $30 \mathrm{mg}$ TAM) were added to each cell (containing $900 \mathrm{~mL}$ physiological saline at $37 \pm 1^{\circ} \mathrm{C}$ and $50 \mathrm{rpm}$ ). TAM concentrations were measured using a UV-Vis spectrophotometer (UV-2102PC, Unico Instrument Co., Ltd., Shanghai, China) under its absorbance at $\lambda=278 \mathrm{~nm}$. All experiments were repeated six times.

\section{Results and Discussion}

3.1. Designing of Modified Coaxial Electrospinning. The common sense about coaxial electrospinning is that the simultaneous treatment of double fluids in an inner-outer manner would generate core-sheath nanostructures, which were duplicated from a concentric spinneret. Switching off one of the double fluids, the concentric spinneret is able to implement a traditional 1-fluid blending electrospinning. As shown in Figure 1(a), the key element in the implementation is the sheath working fluid. When it is electrospinnable, the process is a typical traditional coaxial process, by which coresheath nanofibers are generated. When it is unspinnable, the process is a modified coaxial process. And a special example of the unspinnable fluid is the pure solvent, by which uniform nanofibers can be produced from the coaxial process.

In the coaxial electrospinning system, the key element is the concentric spinneret. A diagram is shown in Figure 1(b).
A capillary is nested into another capillary to form a concentric nozzle, by which the double working fluids are guided into the electrical field. Often, the inner capillary projects slightly (here is $0.2 \mathrm{~mm}$ ) over the outer capillary for easy envelopment of the core solution by the sheath fluid during the electrospinning processes.

In this study, the drug-polymer blending solutions were pumped into the concentric spinneret through a silicon tube, whereas the syringe containing sheath ethanol was directly connected with the spinneret. The electrostatic energy was introduced into the working fluids through an alligator clip, as indicated in Figure 2(a). With methylene blue as a color marker, the modified coaxial processes can be clearly captured by a camera in Figure 2(b). When a high voltage of $12 \mathrm{kV}$ was applied on the initial droplet for generating nanofibers F2, a typical compound Taylor cone was generated (the bottom-right inset), followed by a straight fluid jet and a gradually enlarged blending and whipping unstable region.

3.2. The Morphology of the Prepared 4th SDs. The SEM images of the surface morphologies of nanofibers F1 and F2 are shown in Figures 3(a) and 3(b), respectively. Both of them had a "linear" morphology without any discerned beads or spindles. Their cross sections are shown in Figures 3(c) and 3(d), respectively, which suggested that no solid phase separations occurred during the electrospinning processes, regardless of the single-fluid or the modified coaxial processes.

A comparison between nanofibers F1 and F2 suggests that the latter had a better quality than the former in terms of their sizes and size distributions. As shown in Figure 4, nanofibers F1 had an average diameters of $870 \pm 230 \mathrm{~nm}$ (Figure 4(a)), whereas nanofibers F2 had average diameters of $330 \pm 50 \mathrm{~nm}$ (Figure 4(b)). The nanofibers F2 had a smaller diameter and a narrower size distribution than $\mathrm{F}$. The surrounding sheath solvent ethanol not only made the core blending solutions be 


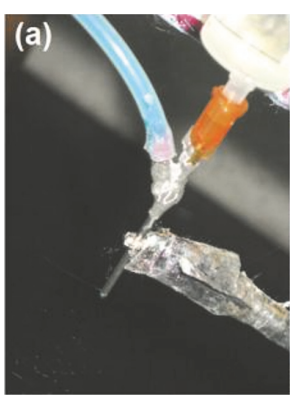

(a)

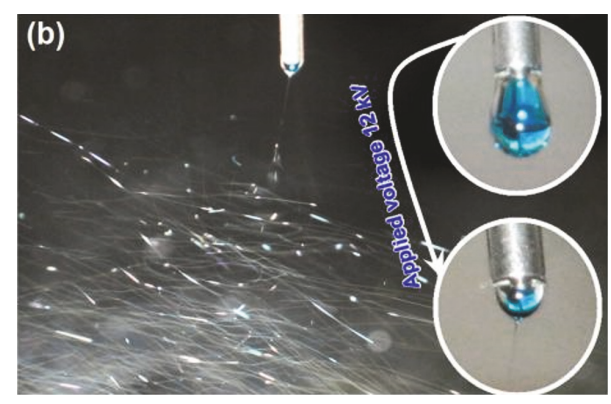

(b)

FIGURE 2: Digital pictures about the connections of spinneret (a) and a typical working process of the modified coaxial electrospinning (b).

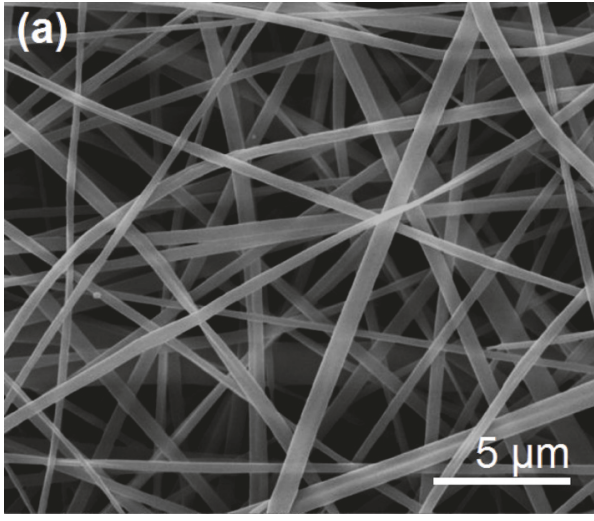

(a)

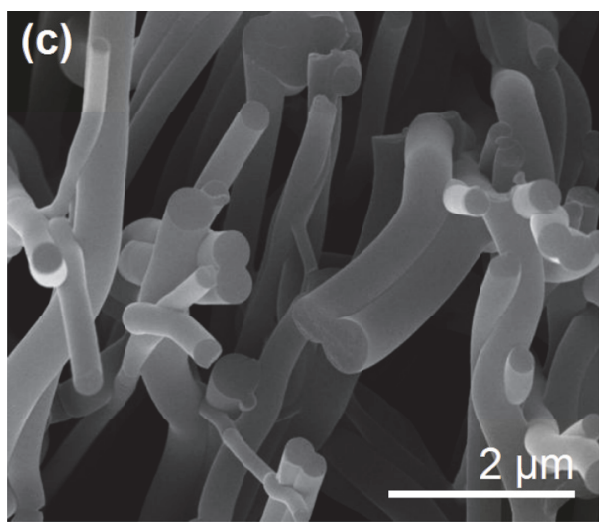

(c)

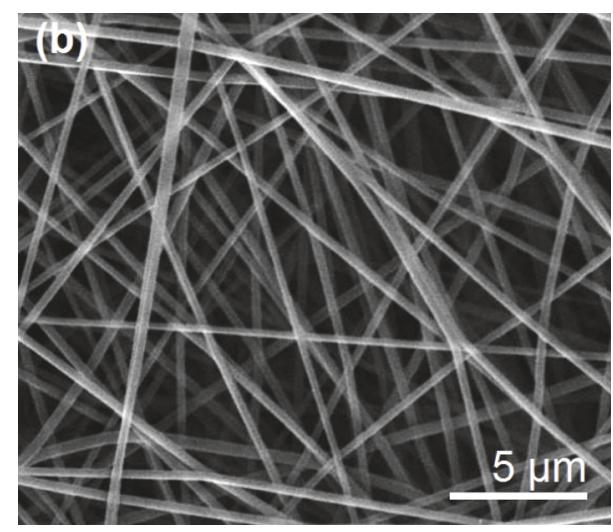

(b)

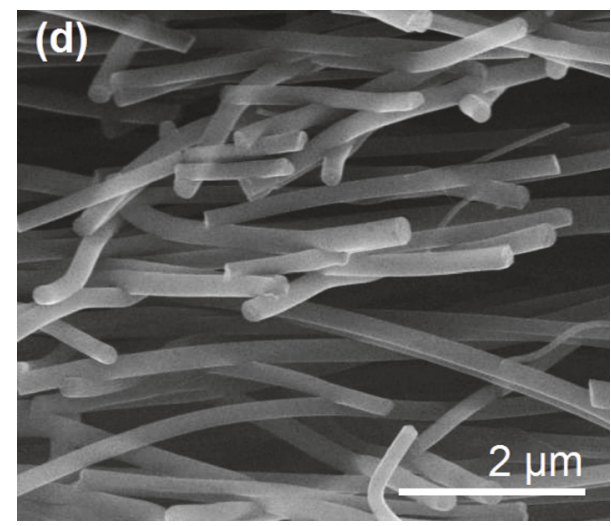

(d)

Figure 3: SEM images about the surface morphologies of nanofibers F1 (a) and nanofibers F2 (b), and their cross sections (c) and (d), respectively.

drawn under the electrical field for a longer time period to reduce their sizes, but also resisted the possible negative influences from the environment to generate nanofibers stably and robustly.

3.3. XRD Characterization and Compatibility. The raw TAM powders and PEG thin slices are crystalline materials, as suggested by their sharp peaks in their XRD patterns (Figure 5).
In contrast, EC is an amorphous polymeric carrier. After being treated by the two electrospinning processes, the working fluids were similarly converted into solid nanofibers, which were amorphous with two halos at their XRD patterns. These suggested that both the raw crystalline drug and PEG were distributed into the EC matrix in a molecular manner in nanofibers F2, and similarly TAM is distributed in nanofibers F1. 


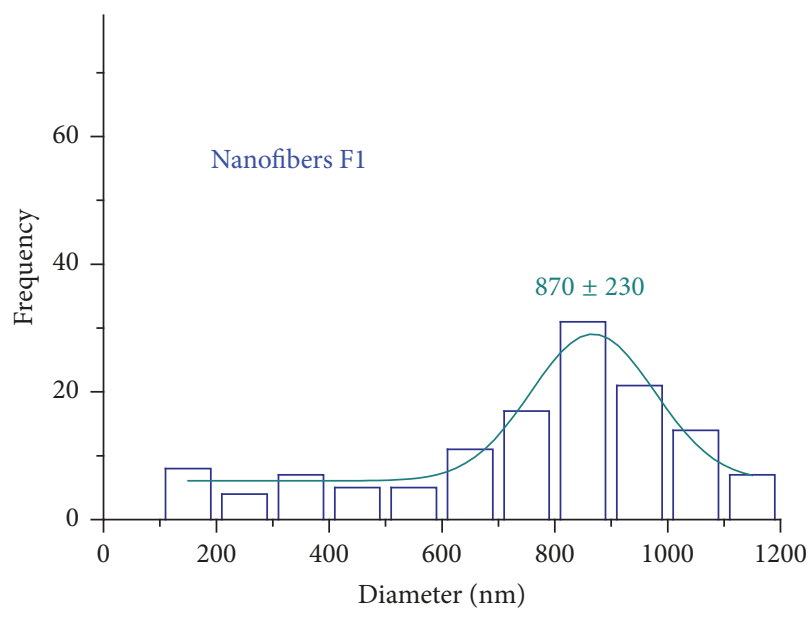

(a)

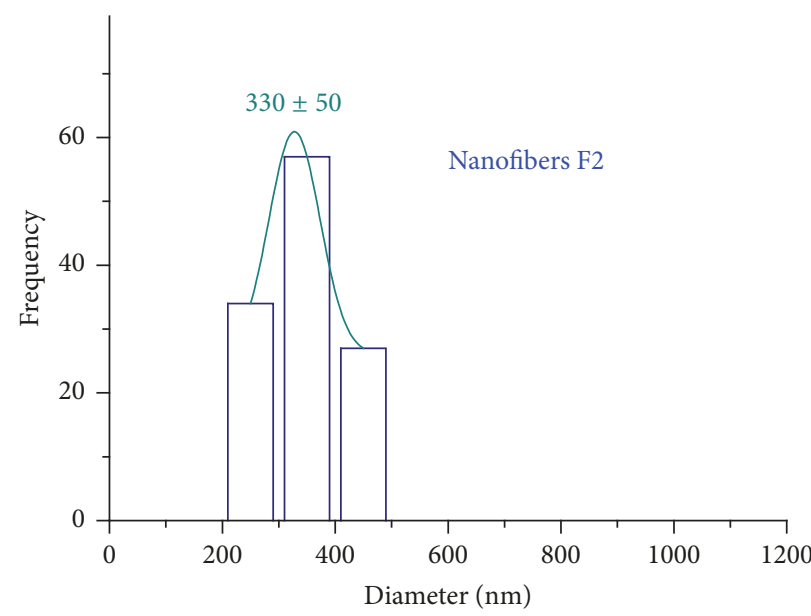

(b)

FIGURE 4: Average diameters and size distributions of nanofibers F1 (a) and nanofibers F2 (b).

TABLE 1: Comparison of the release parameters of nanofibers from different processes ${ }^{\mathrm{a}}$.

\begin{tabular}{lllrr}
\hline \multirow{2}{*}{ Nanofibers } & \multicolumn{4}{c}{ Time needed for releasing a certain percentage of TAM (h) } \\
& $t_{30 \%}$ & $t_{50 \%}$ & $t_{80 \%}$ & $t_{90 \%}$ \\
\hline F1 & 0.95 & 2.04 & 9.23 & 15.24 \\
F2 & 0.87 & 2.62 & 8.67 & 28.34 \\
\hline
\end{tabular}

${ }^{\mathrm{a}} t_{x \%}$ means the time needed for releasing $x \%$ of the contained TAM in the nanofibers.

Compatibility between the drug and its carrier is crucial for the stability and practical applications of electrospun SDs. Therefore, ATR-FTIR experiments were conducted to detect the potential secondary interactions among the components. The spectra of the raw materials and their electrospun 4 th SDs are shown in Figure 6. The raw TAM powders had characteristic peaks at 1724,1581 , and $1508 \mathrm{~cm}^{-1}$; these peaks were attributed to carbonyl groups and benzene rings. However, these typical peaks and also those presented in the finger regions of TAM disappeared from the spectra of nanofibers F1 and F2. These disappearances demonstrated that no TAM crystalline lattices existed in nanofibers F1 and F2 and also no PEG crystalline lattices existed in nanofibers F2, revealing the fine compatibility among these components.

The molecular formulas of EC, PEG, and TAM are shown in Figure 6(b). TAM molecules have both active protons and carbonyl groups. Thus, they can form acicular crystals as the crude particles through hydrogen bondings (Figure 6(c)). When TAM, EC, and PEG were electrospun into nanofibers from their codissolving solutions, $\mathrm{EC}$ and PEG molecules can provide $-\mathrm{OH}$ groups to form hydrogen bonds with $-\mathrm{C}=\mathrm{O}$ groups from TAM molecules. This is favorable to prevent the formation of TAM crystal nucleus and keep its amorphous state in the nanofiber-based SDs.
3.4. In Vitro Dissolution. Figure 7(a) and Table 1 show the drug sustained-release properties of TAM from the two SDs. Both of them provided a certain drug sustained-release time period with an initial burst release effect. However, binary nanofibers $\mathrm{F} 1$ released $91.1 \%$ and $95.7 \%$ of the loaded cargoes after $16 \mathrm{~h}$ and $30 \mathrm{~h}$. In contrast, ternary nanofibers $\mathrm{F} 2$ released 98.6\% and $100.3 \%$ TAM after $16 \mathrm{~h}$ and $24 \mathrm{~h}$. Particularly, $15.24 \mathrm{~h}$ and $28.34 \mathrm{~h}$ were needed for nanofibers $\mathrm{F} 1$ to release $90 \%$ and $95 \%$ of the loaded drug, whereas only $9.57 \mathrm{~h}$ and $13.61 \mathrm{~h}$ were needed for nanofibers F2 (Table 1). These results suggested that the nanofibers $\mathrm{F} 1$ had a negative longer time period of leveling-off release and trapped some drug within them, suggesting a poorer drug sustained-release profile than nanofibers F2.

Release data from the SDs can be analyzed according to the power law expression shown in the following equation. The kinetics and mechanism of drug release for each nanofiber could be disclosed by fitting the release data to the following [22]:

$$
\begin{aligned}
Q & =\frac{M_{T}}{M_{\infty}}=k T^{n}, \\
\log Q & =n \log (T)+\log (k),
\end{aligned}
$$

where $M_{T}$ is the drug released amount at time $T, M_{\infty}$ is the drug released amount finally, $k$ is the kinetic constant, 

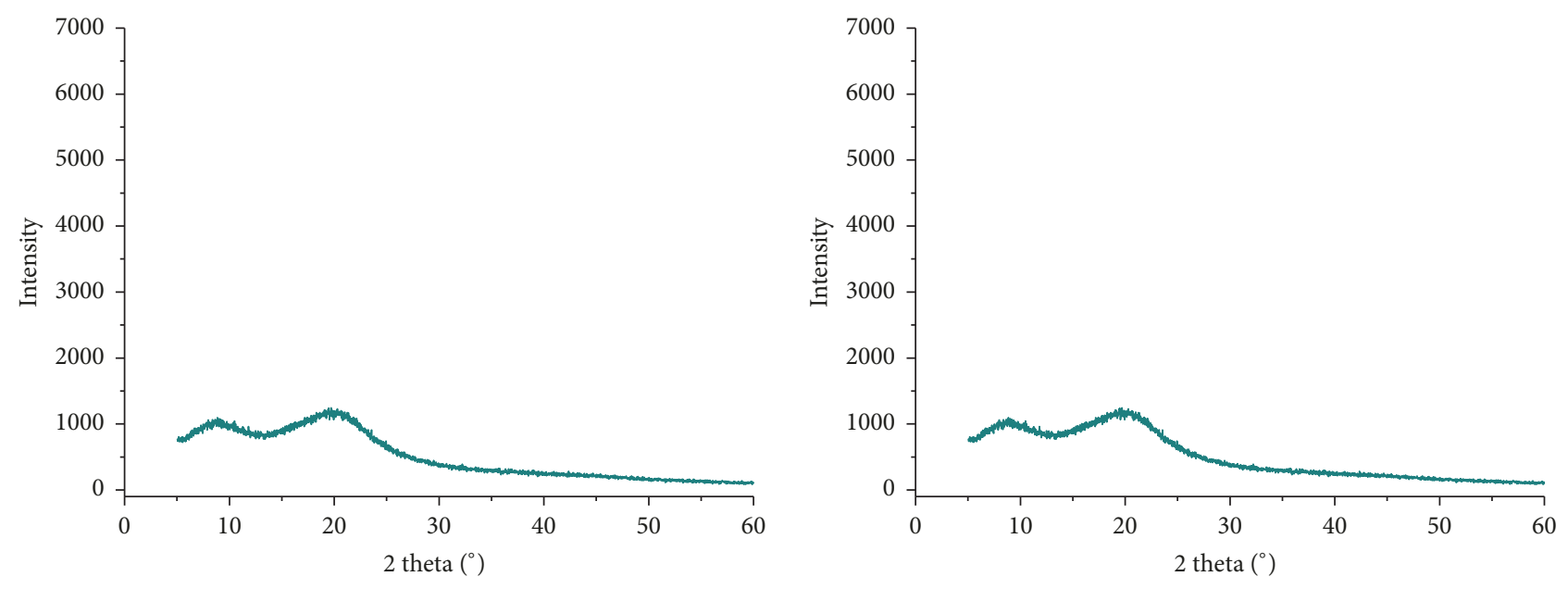

- B

$$
-\mathrm{B}
$$

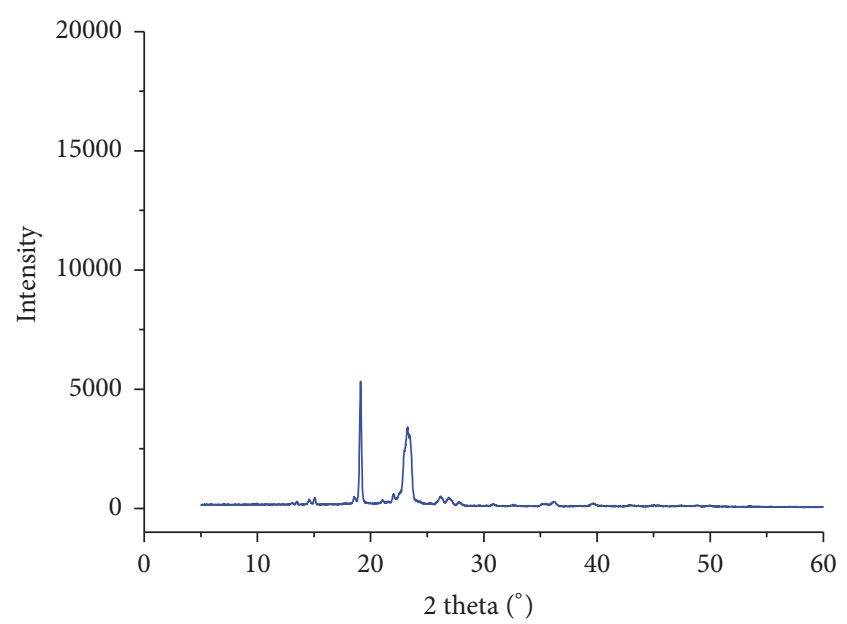

$-\mathrm{B}$

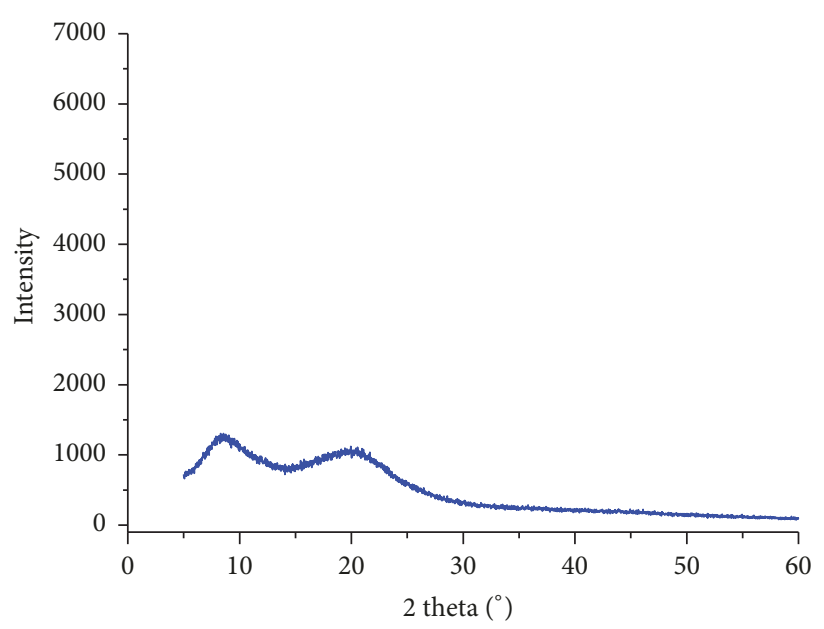

- B

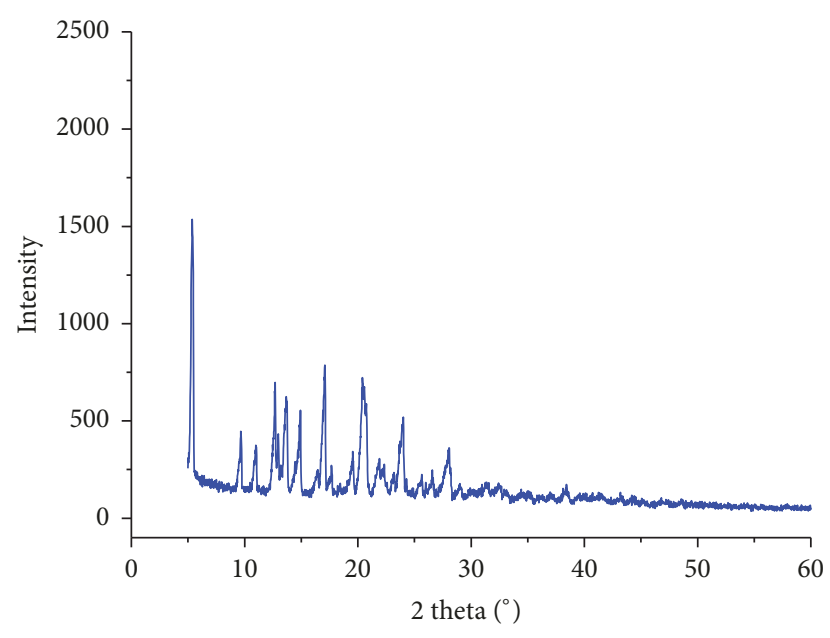

B

FIGURE 5: XRD patterns of the raw materials and their 4th SDs from different processes. 

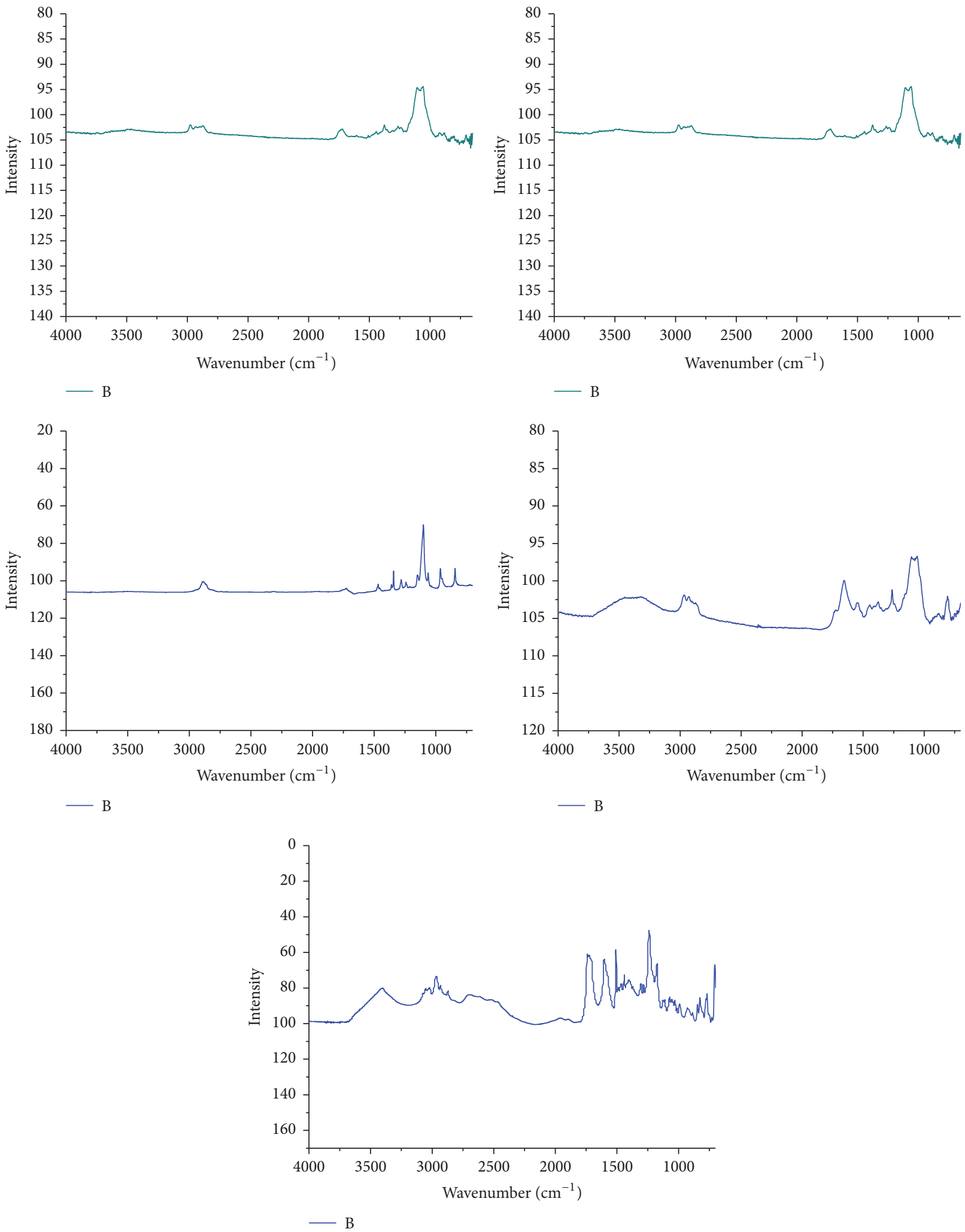

(a)

FIgURE 6: Continued. 


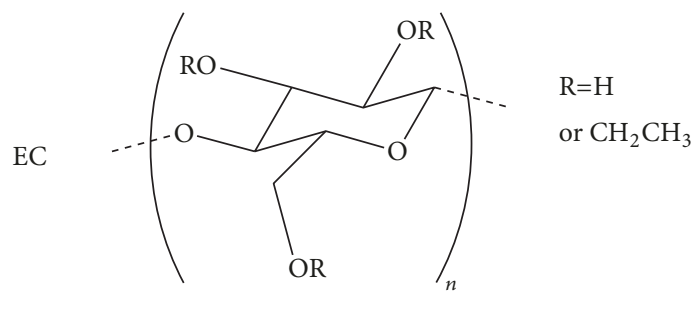

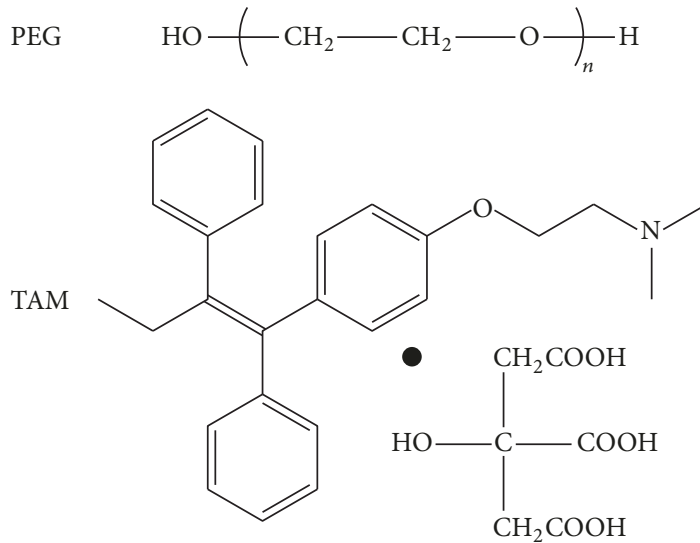

(b)

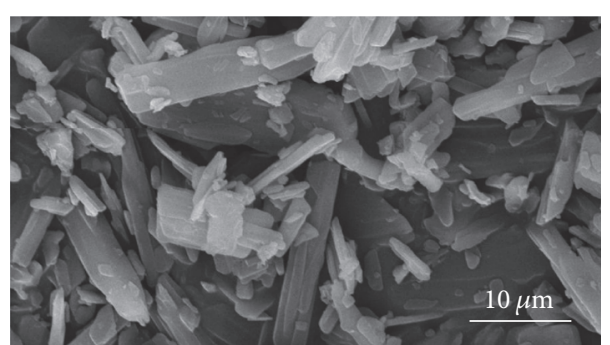

(c)

FIGURE 6: (a) FTIR spectra of the crude materials and their 4th SDs from different processes; (b) molecular formula of EC, PEG, and TAM; (c) SEM images of crude TAM particles.

and $n$ is the release exponent. The two 4th SDs have the following regressed equations (Figure 7(b)): $\log Q_{1}=1.54+$ $0.37 \log T_{1}(R=0.9807) ; \log Q_{2}=1.55+0.37 \log T_{2}(R=$ $0.9988)$. It is obvious that both SDs freed their loaded cargoes through a typical Fickian diffusion mechanism because their diffusion index $n$ had a value smaller than 0.45 . The additives of a small content of soluble PEG did not change the drug diffusion release mechanism from the insoluble skeleton EC.

EC is frequently utilized in pharmaceutical industry and also laboratories as polymer skeleton for giving drug sustained-release profiles in the forms of tablets, nanoparticles, coating membranes, and casting films [23]. One concern is that the encapsulated drug is often not able to be totally freed out, just as here the nanofibers F1 having a release of $95.7 \%$ after $32 \mathrm{~h}$. The incorporation of PEG into the EC skeleton could effectively alter the drug release behaviors; a diagram is shown in Figure 8. When nanofibers F2 were immersed in the dissolution media, the soluble oligomer PEG would first dissolve into the penetrated water and diffuse out from the fibers to the bulk dissolution media. Meanwhile, some poorly water-soluble TAM molecules would also free into the dissolution media due to their molecular distribution and also their surface distribution around the fibers. The dissolved PEG molecules would leave interconnected holes within the insoluble EC skeleton in the fibers. Later, the remained TAM molecules would take these holes as ways to diffuse to the outer media. Thus, the synergistic effects of EC (as insoluble skeleton for a diffusion mechanism) and PEG (as a hole-making agent for promoting drug molecule diffusion) in nanofibers F2 jointly manipulated the TAM molecules release behaviors and released all the loaded molecules with little late leveling-off release effect, showing better functional performances than their binary counterparts F1.

\section{Conclusions}

A novel 4th SD in the form of electrospun nanofibers consisting of three components with each having its special function was successfully fabricated using a modified coaxial electrospinning process. Compared with its counterpart, the binary 4th SD composed of TAM and EC created using a 1-fluid blending process, the new SD had higher quality in terms of the diameter and the related size distribution. Although both 4th SDs were amorphous nanocomposites due to the fine compatibility among the components, nanofibers F2 could provide a better drug sustained-release profile than nanofibers F1. The ternary 4th SDs could exhaust all the loaded cargoes within a certain time period with little late leveling-off release effect, suggesting their potential applications as oral drug delivery systems for furnishing drug sustained-release profiles. Based on the applications of advanced modified coaxial electrospinning, the present work showed a new strategy for developing novel functional nanomaterials.

\section{Conflicts of Interest}

The authors declare that they have no conflicts of interest. 


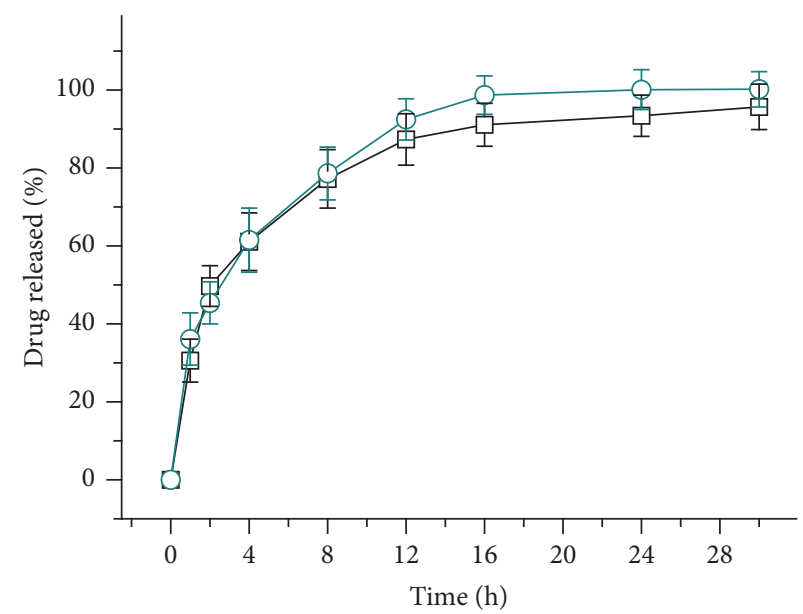

$-\square-$ Nanofibers F1

-O- Nanofibers F2

(a)

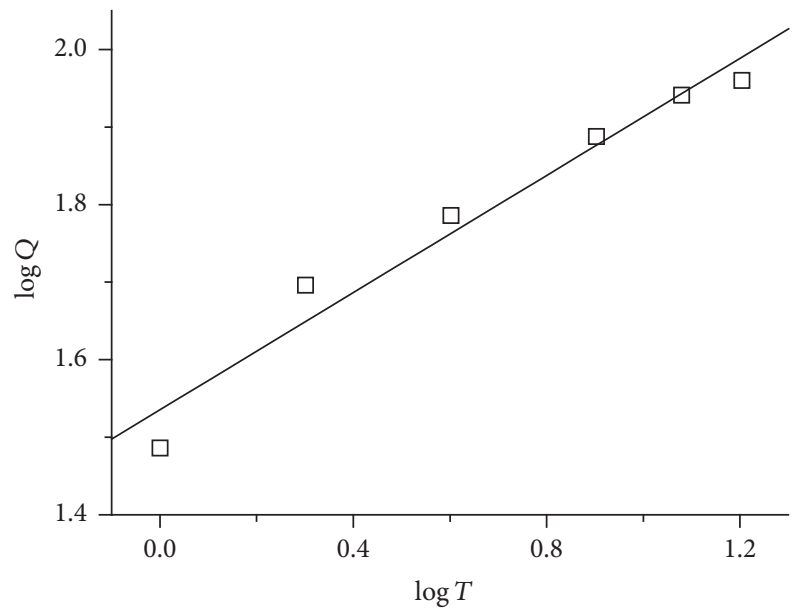

$-\square$ Nanofibers F1

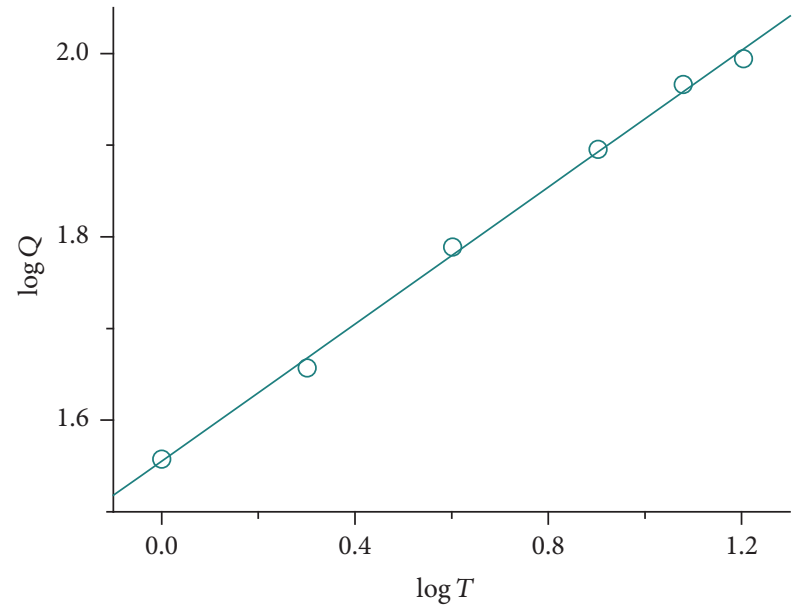

-O- Nanofibers F2

(b)

Figure 7: In vitro release profiles of TAM (a) and Peppas equation regressed results (b).

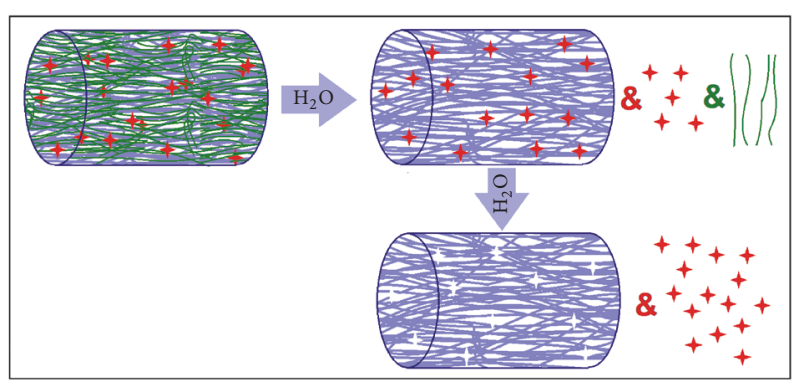

ICON

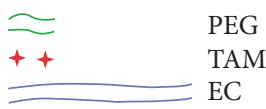

FIGURE 8: A diagram showing the drug sustained-release mechanism from the ternary 4 th SD nanofibers F2.

\section{Authors' Contributions}

Zhu Zhang and Wenbing Li contributed equally to this work.

\section{Acknowledgments}

The National Natural Science Foundation of China (no. 51373101), the College Student Innovation Project (nos. USST20170286 and SH2017189.190.191), and China Scholarship Council Grant (no. 201708420212) are highly appreciated.

\section{References}

[1] Y. Xu, J.-J. Li, D.-G. Yu, G. R. Williams, J.-H. Yang, and X. Wang, "Influence of the drug distribution in electrospun gliadin fibers on drug-release behavior," European Journal of Pharmaceutical Sciences, vol. 106, pp. 422-430, 2017. 
[2] B. Démuth, Z. K. Nagy, A. Balogh et al., "Downstream processing of polymer-based amorphous solid dispersions to generate tablet formulations," International Journal of Pharmaceutics, vol. 486, no. 1-2, pp. 268-286, 2015.

[3] D. D. Sun and P. I. Lee, "Probing the mechanisms of drug release from amorphous solid dispersions in medium-soluble and medium-insoluble carriers," Journal of Controlled Release, vol. 211, pp. 85-93, 2015.

[4] C. Vo, C. Park, and B. Lee, "Current trends and future perspectives of solid dispersions containing poorly water-soluble drugs," European Journal of Pharmaceutics and Biopharmaceutics, vol. 85, pp. 799-813, 2013.

[5] Z. Iqbal, A. Babar, and M. Ashraf, "Controlled-release naproxen using micronized ethyl cellulose by wet-granulation and soliddispersion method," Drug Development and Industrial Pharmacy, vol. 28, no. 2, pp. 129-134, 2002.

[6] Y. Yang, M. Zhang, Z. Liu, K. Wang, and D. Yu, "Meletin sustained-release gliadin nanoparticles prepared via solvent surface modification on blending electrospraying," Applied Surface Science, vol. 434, pp. 1040-1047, 2018.

[7] Z. Khatri, I.-S. Kim, and S. H. Kim, "Functional nanofibers: production and applications," Journal of Nanomaterials, vol. 2016, Article ID 2195787, pp. 1-2, 2016.

[8] U. A. Qureshi, Z. Khatri, F. Ahmed, M. Khatri, and I.-S. Kim, "Electrospun Zein Nanofiber as a Green and Recyclable Adsorbent for the Removal of Reactive Black 5 from the Aqueous Phase," ACS Sustainable Chemistry \& Engineering, vol. 5, no. 5, pp. 4340-4351, 2017.

[9] A. W. Jatoi, Y. K. Jo, H. Lee et al., "Antibacterial efficacy of poly(vinyl alcohol) composite nanofibers embedded with silver-anchored silica nanoparticles," Journal of Biomedical Materials Research Part B: Applied Biomaterials, 2017.

[10] K. Zhang, W. Yuan, N. Zhou, and C. Wu, "Multicomponent nanofibers via electrospinning of polymers and colloidal dispersions for environmental and optical applications," in Electrospun Nanofibers for Energy and Environmental Applications, B. Ding and J. Yu, Eds., Nanostructure Science and Technology, pp. 403431, Springer, Berlin, Germany, 2014.

[11] W. Yuan and K.-Q. Zhang, "Structural evolution of electrospun composite fibers from the blend of polyvinyl alcohol and polymer nanoparticles," Langmuir, vol. 28, no. 43, pp. 1541815424, 2012.

[12] E.-R. Kenawy, G. L. Bowlin, K. Mansfield et al., "Release of tetracycline hydrochloride from electrospun poly(ethyleneco-vinylacetate), poly(lactic acid), and a blend," Journal of Controlled Release, vol. 81, no. 1-2, pp. 57-64, 2002.

[13] X.-F. Liu, Y.-K. Lai, J.-Y. Huang, S. S. Al-Deyab, and K.-Q. Zhang, "Hierarchical SiO2@Bi2O3 core/shell electrospun fibers for infrared stealth camouflage," Journal of Materials Chemistry C, vol. 3, no. 2, pp. 345-351, 2015.

[14] D. Yu, J. Li, M. Zhang, and G. R. Williams, "High-quality Janus nanofibers prepared using three-fluid electrospinning," Chemical Communications, vol. 53, no. 33, pp. 4542-4545, 2017.

[15] K. Wang, X. K. Liu, X. H. Chen, D. G. Yu, Y. Y. Yang, and P. Liu, "Electrospun hydrophilic Janus nanocomposites for the rapid onset of therapeutic action of helicid," ACS Applied Materials \& Interfaces, vol. 10, no. 3, pp. 2859-2867, 2018.

[16] C. Sealy, "Nanoscale fibers get drug delivery just right," Materials Today, vol. 20, no. 4, p. 160, 2017.

[17] C. Sealy, "New spin on drug-loaded composite fibers," Materials Today, vol. 19, no. 7, p. 374, 2016.
[18] Z. K. Nagy, A. Balogh, B. Démuth et al., "High speed electrospinning for scaled-up production of amorphous solid dispersion of itraconazole," International Journal of Pharmaceutics, vol. 480, no. 1-2, pp. 137-142, 2015.

[19] Q. Wang, D.-G. Yu, L.-L. Zhang, X.-K. Liu, Y.-C. Deng, and M. Zhao, "Electrospun hypromellose-based hydrophilic composites for rapid dissolution of poorly water-soluble drug," Carbohydrate Polymers, vol. 174, pp. 617-625, 2017.

[20] Z. Liu, Y. Zhang, D. Yu, D. Wu, and H. Li, "Fabrication of sustained-release zein nanoparticles via modified coaxial electrospraying," Chemical Engineering Journal, vol. 334, pp. 807-816, 2018.

[21] T. Vasconcelos, B. Sarmento, and P. Costa, "Solid dispersions as strategy to improve oral bioavailability of poor water soluble drugs," Drug Discovery Therapy, vol. 12, no. 23-24, pp. 10681075, 2007.

[22] N. A. Peppas, "Analysis of Fickian and non-Fickian drug release from polymers," Pharmaceutica Acta Helvetiae, vol. 60, no. 1, pp. 110-111, 1985.

[23] X. Li, Z. Zheng, D. Yu, X. Liu, Y. Qu, and H. Li, “Electrosprayed sperical ethylcellulose nanoparticles for an improved sustainedrelease profile of anticancer drug," Cellulose, vol. 24, no. 12, pp. 5551-5564, 2017. 


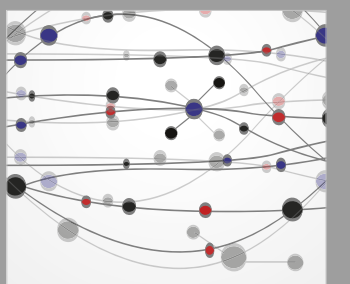

The Scientific World Journal
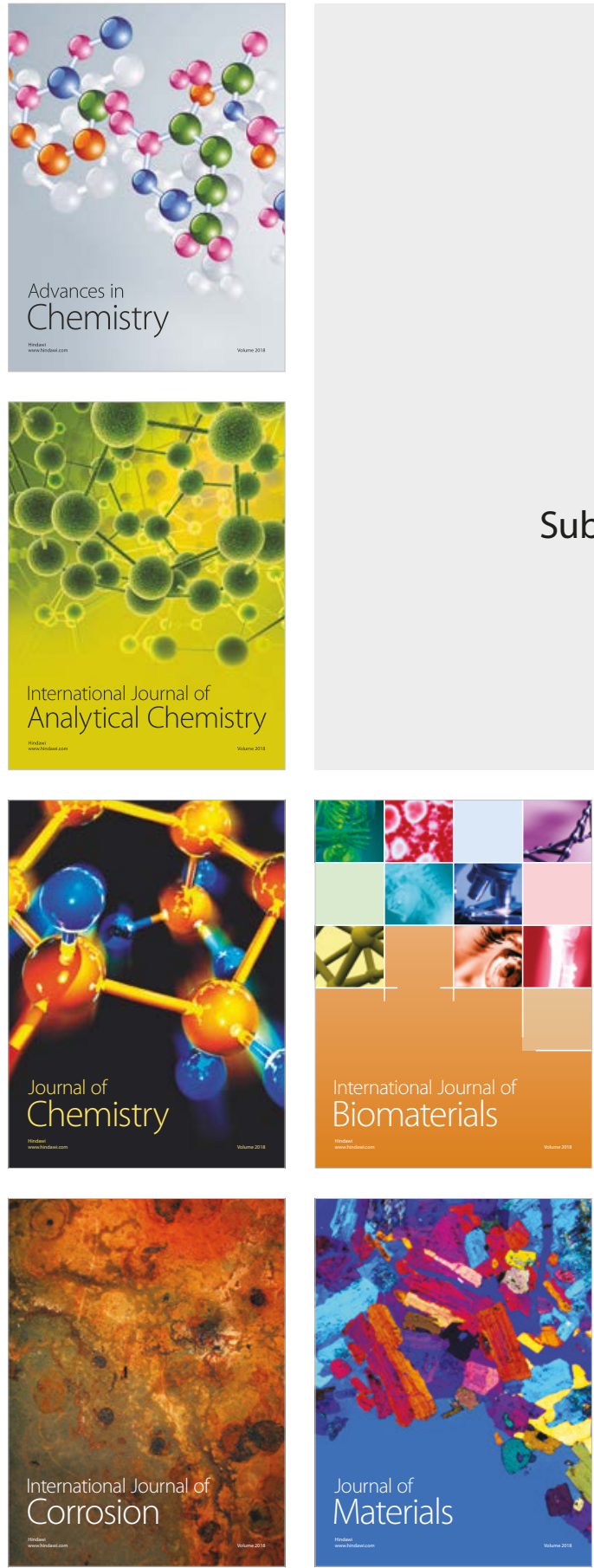

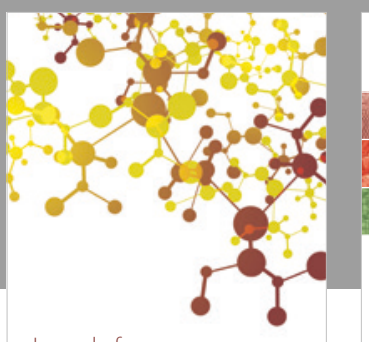

Journal of

Applied Chemistry
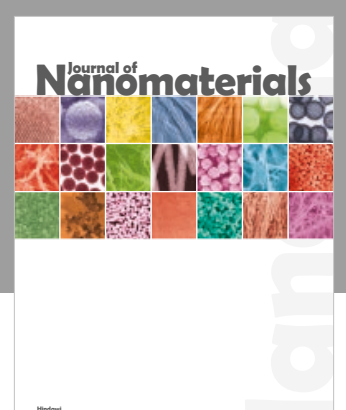

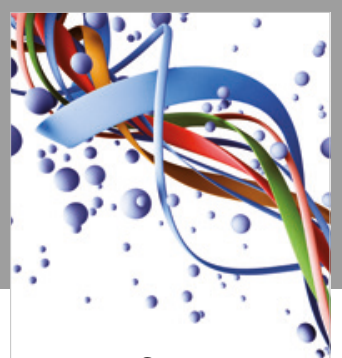

Scientifica

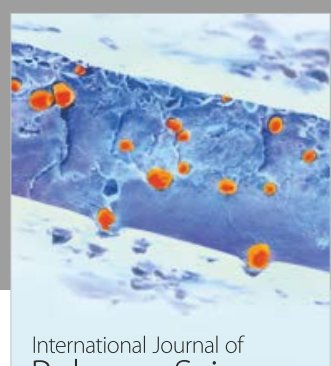

Polymer Science

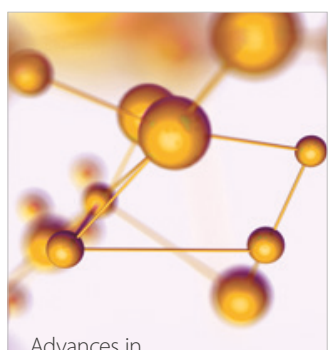

Physical Chemistry
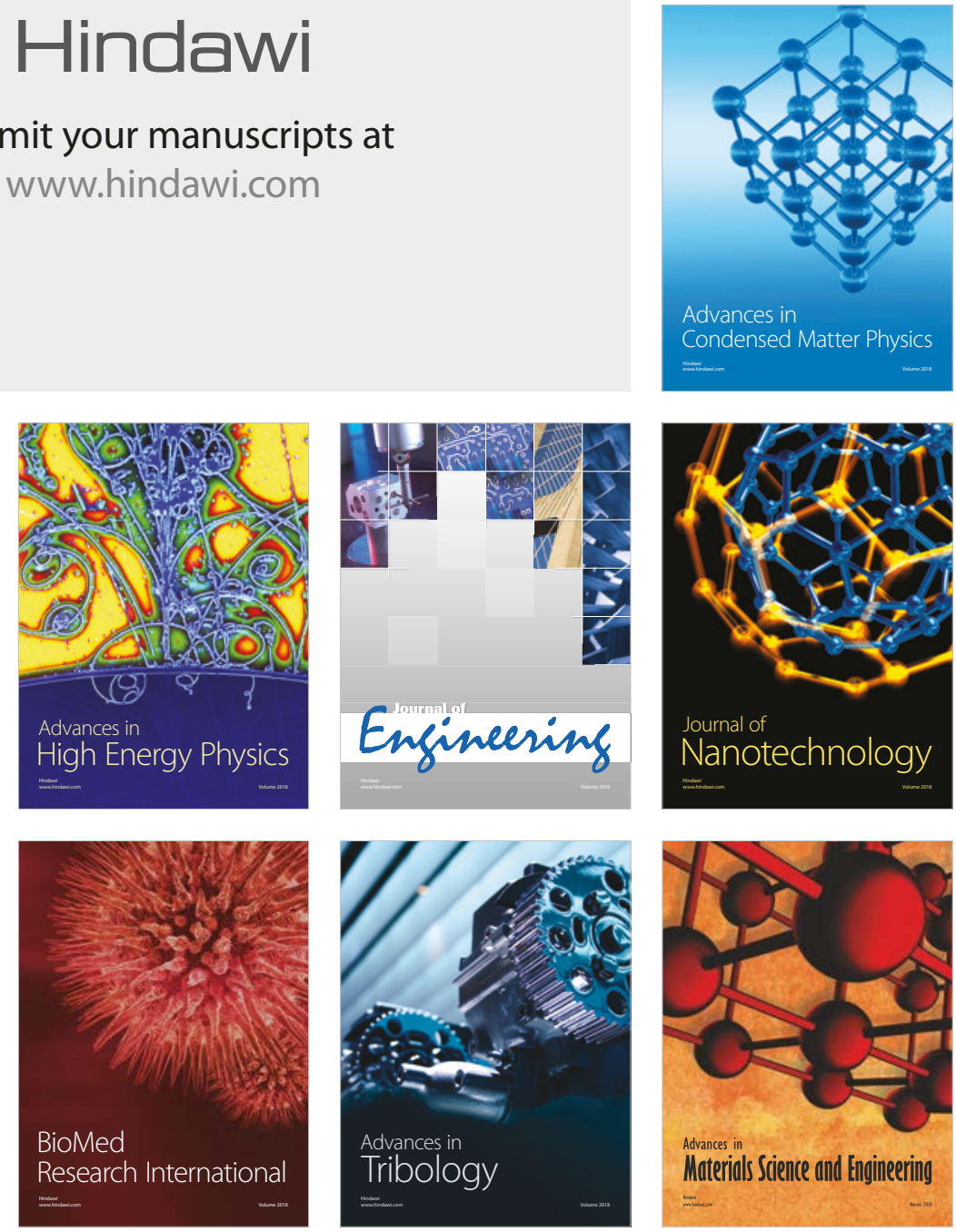